\title{
IOGURTES COM POLPA DE NONI E ACEROLA: AVALIAÇÃO FÍSICO-QUÍMICA, ATIVIDADE ANTIOXIDANTE E PERFIL SENSORIAL
}

\author{
Andrezza Assis Cruz Moura* \\ Edna Maria Mendes Aroucha** \\ Vílson Alves de Góis ${ }^{* * *}$ \\ Ricardo Henrique de Lima Leite ${ }^{* *+* t}$ \\ Rafaella Martins de Araújo Ferreira*t+** \\ Mônica Cristina de Paiva Silva*
}

\begin{abstract}
A inovação na elaboração de produtos pela indústria de alimentos visa agregar atributos de qualidade diferencial, tal como propriedades antioxidantes. Assim, no desenvolvimento de novos sabores de iogurtes de frutas a avaliação sensorial é necessária para assegurar uma boa comercialização. Dessa forma, o objetivo deste estudo foi elaborar iogurtes com polpa de noni e acerola e avaliar as propriedades físico-químicas, atividade antioxidante e perfil sensorial. O iogurte natural foi utilizado como controle $(0: 0)$ e os demais tratamentos foram obtidos com as proporções de polpa de noni e acerola: $(0: 2,5) ;(2,5: 0) ;(1,25: 1,25) ;(0: 5) ;(5: 0)$ e $(2,5: 2,5)$. A acidez titulável, gordura e proteína lácteas, diferiram com o tratamento, mas permaneceram dentro dos padrões de qualidade estabelecidos pela legislação brasileira. $O$ tratamento (0:5) apresentou maior teor de fenólicos e junto com o tratamento $(0: 2,5)$ apresentaram maior atividade antioxidante. Com exceção do tratamento (5:0), os demais iogurtes apresentaram aceitação e intenção de compra semelhante ao iogurte natural. $O$ tratamento $(2,5: 0)$ apresentou menor rejeição pelo teste de intenção de compra. Assim, todos os iogurtes elaborados possuem grande potencialidade comercial.
\end{abstract}

PALAVRAS-CHAVE: LEITE, NONI, FENÓLICOS, ACEROLA.

\footnotetext{
* Mestrandas em Produção Animal, Universidade Federal Rural do Semi-Árido (UFERSA), Mossoró, RN (e-mail: andrezzabarreto@ufersa.edu.br; monica.silva-2009@hotmail.com).

** Doutora em Produção Vegetal, Professora, Departamento de Agrotecnologia e Ciências Sociais, Universidade Federal Rural do Semi-Árido (UFERSA), Mossoró, RN (e-mail: aroucha@ufersa.edu.br).

*** Doutor em Fitotecnia, Professor, Departamento de Agrotecnologia e Ciências Sociais, Universidade Federal Rural do Semi-Árido (UFERSA), Mossoró, RN (e-mail: vilsongois@ufersa.edu.br).

****Doutor em Química Analítica, Professor, Departamento de Agrotecnologia e Ciências Sociais, Universidade Federal Rural do Semi-Árido (UFERSA), Mossoró, RN (e-mail: ricardoleite@ufersa.edu.br).

***** Doutoranda em Fitotecnia, Universidade Federal Rural do Semi-Árido (UFERSA), Mossoró, RN (e-mail: rafaellamarafe@gmail.com).
} 


\section{INTRODUÇÃO}

O iogurte se destaca pelo seu valor nutritivo, digestibilidade e benefícios à saúde, e por ser considerado um alimento probiótico (THAMER \& PENNA, 2006), pois contém em sua formulação bactérias vivas e ativas. Além disso, tem grande aceitabilidade, pois agrada todas as faixas etárias e possui baixo custo. No Brasil, o consumo de iogurte cresceu consideravelmente nos últimos anos, aumentando de 3,5\% em 2007 (NIELSEN, 2007) para 13\% em 2011 (NIELSEN, 2011).

A procura por alimentos com qualidade, praticidade, propriedades funcionais, segurança e preços acessíveis vem crescendo e, assim, as inovações na elaboração dos produtos é objetivo constante na indústria de alimentos, que visa agregar atributos de qualidade diferencial ao produto. Nesse sentido, o mercado que oferta produtos com formulação mista, ou seja, de origem animal e vegetal, tem grande comercialização, como os iogurtes elaborados com polpas ou geléias de frutas (GIESE, 2010).

$\mathrm{Na}$ busca de alimentos com alguma funcionalidade para o organismo, o fruto noni, cuja presença expressiva de atividade antioxidante, devido a presença de fenólicos e carotenóides (CANUTO et al., 2010), surge como um alimento bem consumido. Da mesma forma, a acerola também tem se destacado com grande aceitação e elevado teor de vitamina C (ASSIS et al., 2001), que a faz uma ótima fonte de antioxidantes.

Tanto os compostos fenólicos (ESCARPA \& GONZÁLEZ, 2001) quanto a vitamina C (FENNEMA, 2000) desempenham funções relevantes no corpo humano, pois atuam reduzindo e prevenindo o acúmulo de produtos tóxicos resultantes do metabolismo celular, ou seja, são antioxidantes (ISHIKAWA \& SHIGEOKA, 2008) e, atualmente, esses alimentos com propriedades funcionais possuem grande apelo para sua aquisição no mercado, com maior valor agregado.

Em uma linha de produção, o desenvolvimento de novos sabores de iogurtes vem acompanhado de uma série de testes (LIMA et al., 2003) para então serem comercializados. Devem apresentar características físico-químicas conforme estabelece a legislação brasileira pela Instrução Normativa $n^{\circ} 46$ (BRASIL, 2007). Todavia, os testes sensoriais (cor, aroma, sabor, consistência, preferência, intenção de compra) (LOURES et al., 2010) são importantes ferramentas para avaliar a aceitação do produto pelos consumidores. Dessa forma, Giese et al. (2010) verificaram em seus estudos sobre caracterização físico-química e sensorial de iogurtes comerciais que os produtos com menor acidez foram os preferidos pelos consumidores.

Nesse sentido, sabendo-se que as polpas possuem peculiaridades distintas e, que a sua adição ao iogurte pode modificar a sua qualidade e aceitação. Este trabalho teve por objetivo avaliar algumas propriedades físico-químicas, antioxidantes e sensoriais de iogurte elaborado com polpa de noni (Morinda citrifolia L.) e acerola (Malpighia emarginata DC.).

\section{MATERIAL E MÉTODOS}

\subsection{PROCESSO DE FABRICAÇÃO DOS IOGURTES}

A fabricação do iogurte bem como a introdução da polpa dos frutos foi elaborada conforme procedimento descrito por Ordóñez (2005) e apresentada na Figura 1.

\subsection{ELABORAÇÃO DOS IOGURTES ACRESCIDOS DAS POLPAS}

As polpas foram extraídas de frutos na maturação comercial. Após lavagem e sanitização, foram processadas e acondicionadas em recipientes plásticos fechados e mantidas sob refrigeração a $8^{\circ} \mathrm{C}$ até o momento da elaboração dos iogurtes. 
O iogurte natural foi considerado como controle $(0,0)$ e os demais tratamentos formulados com adição de polpas em diferentes proporções de noni: acerola foram os seguintes: (0:2,5); (2,5:0); $(1,25: 1,25) ;(0: 5) ;(5: 0)$ e $(2,5: 2,5)$.

Após a elaboração dos iogurtes com as diferentes concentrações de polpa dos frutos, os mesmos foram avaliados quanto às propriedades físico-químicas e antioxidantes e, após sete dias de refrigeração, efetuou-se a análise sensorial.

\subsection{ANÁLISES FÍSICO-QUÍMICAS E ATIVIDADE ANTIOXIDANTE}

As análises descritas a seguir foram realizadas nos produtos em três repetições (lotes) e, para cada repetição, foi realizada análises em triplicatas. $\mathrm{pH}$ : utilizou-se potenciômetro modelo Tec-3MP devidamente calibrado, utilizando duas soluções padrão de $\mathrm{pH}=4,0$ e 7,0, conforme Instituto Adolfo Lutz (2008). Acidez titulável: foi realizada por titulometria, conforme recomendação do Instituto Adolfo Lutz (2008). Gordura láctea: determinada com auxílio de butirômetro, conforme recomendações do Instituto Adolfo Lutz (2008). Proteínas lácteas: foi realizada pelo método de micro-Kjeldahl, no qual a proteína bruta foi calculada em função dos teores de nitrogênio total, multiplicado pelo fator 6,38, conforme Instituto Adolfo Lutz (2008). Fenólicos totais: foi realizada conforme método descrito por Meda et al. (2005) utilizando-se o reagente Folin-Ciocalteau (SINGLETON \& ROSSI, 1965). Para os cálculos de fenólicos totais, foi utilizada uma curva padrão de ácido gálico (20 a $200 \mathrm{mg} / \mathrm{L}$ ), os resultados foram expressos em $\mathrm{mg}$ de ácido gálico (AG)/100g de iogurte. Atividade antioxidante: foi realizada com o uso do radical 2,2-difenil-1-picril-hidrazil (DPPH), conforme modificações feitas por Meda et al. (2005), em diluições seriadas. Foram construídas curvas concentração-resposta para cada formulação de iogurte determinando-se o valor do $\mathrm{IC}_{50}$. $\mathrm{A}$ porcentagem de inibição foi calculada e a curva de inibição foi obtida construindo um gráfico com a percentagem de inibição versus a concentração do inibidor (iogurte). Os parâmetros de regressão linear foram traçados para cada curva e os valores de $\mathrm{IC}_{50}$ foram obtidos utilizando o software Microsoft Excel 2007.

\subsection{ANÁLISE SENSORIAL}

Para a análise sensorial participaram 75 provadores não treinados, consumidores de iogurte, de ambos os sexos com faixa etária entre 18 a 60 anos, alunos e funcionários da Universidade Federal Rural do Semi-Árido (UFERSA). Os testes foram realizados no período da manhã de $9 \mathrm{~h}$ às 11h.A pesquisa seguiu as normas vigentes conforme resolução 466/12 do Conselho Nacional de Saúde e foi aprovada pelo Comitê de Ética em Pesquisa da Universidade Estadual do Rio Grande do Norte, conforme parecer 722.498/14.

Cada processamento (lote) de iogurte teve suas análises realizadas por 25 provadores. As amostras foram inicialmente codificadas com três dígitos e para a avaliação foram servidos 20 $\mathrm{mL}$ de cada amostra, em copos plásticos descartáveis, com a temperatura do iogurte em torno de $10^{\circ} \mathrm{C}$, acompanhados com água e biscoito para a limpeza do paladar entre as análises. Utilizou-se escalas hedônicas para os testes de aceitação (1: desgostei muitíssimo a 9: gostei muitíssimo), de consistência (1: pouquíssimo consistente a 9: muitíssimo consistente) e de intenção de compra (1: certamente não compraria a 5: certamente compraria).

\subsection{DELINEAMENTO EXPERIMENTAL E ANÁLISES ESTATÍSTICAS}

O experimento foi conduzido em delineamento inteiramente casualizado (DIC), constando de sete tratamentos. Para tanto, os iogurtes foram formulados três vezes, em semanas consecutivas, sendo cada processamento uma repetição.

Os dados foram submetidos à análise de variância $(p<0,01)$ utilizando o software SISVAR (FERREIRA, 2003). Para a comparação de médias, utilizaram-se os testes de Scott-Knott $(p<0,05)$ e Friedman $(p<0,05)$, respectivamente, para os resultados das análises físico-químicas e sensoriais. 


\section{RESULTADOS E DISCUSSÃO}

\subsection{ANÁLISES FÍSICO-QUÍMICAS E ATIVIDADE ANTIOXIDANTE}

Verificou-se efeito significativo de tratamento para acidez titulável, gordura láctea, proteínas lácteas, teor de fenólicos e atividade antioxidante $\left(\mathrm{IC}_{50}\right)$. Porém, não houve efeito de tratamento para o pH (Tabela 1).

TABELA 1.VALORES MÉdIOS E DESVIOS PADRÕES DE PH, ACIDEZ TITULÁVEL (AT), GORDURA LÁCTEA (GL), PROTEÍNAS LÁCTEAS (PL), TEOR DE FENÓLICOS (TF) E ATIVIDADE ANTIOXIDANTE (AA) DE SETE FORMULAÇÕES DE IOGURTE EM FUNÇÃO DE DIFERENTES CONCENTRAÇÕES DE NONI (MORINDA CITRIFOLIA L.) E ACEROLA (MALPIGHIA EMARGINATA DC.), MOSSORÓ-RN, 2014.

\begin{tabular}{ccccccc}
\hline $\begin{array}{c}\text { Formulação } \\
\text { com } \\
\text { noni:acerola } \\
(\%)\end{array}$ & $\mathrm{pH}$ & $\mathrm{AT}(\%)$ & $\mathrm{GL}(\%)$ & $\mathrm{PL}(\%)$ & $\mathrm{TF}(\mathrm{mg} / 100 \mathrm{~g})$ & $\mathrm{AA}\left(\mathrm{IC}_{50}\right)$ \\
\hline$(0: 0)$ & $4,04 \pm 0,11 \mathrm{a}$ & $0,92 \pm 0,01 \mathrm{a}$ & $3,40 \pm 0,00 \mathrm{a}$ & $2,95 \pm 0,02 \mathrm{~b}$ & $56,42 \pm 5,17 \mathrm{c}$ & $139,40 \pm 4,64 \mathrm{a}$ \\
\hline$(0: 2,5)$ & $4,02 \pm 0,13 \mathrm{a}$ & $0,92 \pm 0,01 \mathrm{a}$ & $3,40 \pm 0,00 \mathrm{a}$ & $2,94 \pm 0,05 \mathrm{~b}$ & $69,47 \pm 4,69 \mathrm{~b}$ & $23,74 \pm 1,65 \mathrm{~d}$ \\
\hline$(2,5: 0)$ & $4,03 \pm 0,12 \mathrm{a}$ & $0,88 \pm 0,01 \mathrm{c}$ & $3,57 \pm 0,06 \mathrm{a}$ & $3,06 \pm 0,03 \mathrm{a}$ & $59,13 \pm 4,27 \mathrm{c}$ & $106,33 \pm 19,09 \mathrm{~b}$ \\
\hline$(1,25: 1,25)$ & $4,06 \pm 0,14 \mathrm{a}$ & $0,90 \pm 0,02 \mathrm{~b}$ & $3,17 \pm 0,06 \mathrm{~b}$ & $2,99 \pm 0,04 \mathrm{~b}$ & $64,69 \pm 4,74 \mathrm{c}$ & $62,86 \pm 9,02 \mathrm{c}$ \\
\hline$(0: 5)$ & $4,02 \pm 0,11 \mathrm{a}$ & $0,93 \pm 0,01 \mathrm{a}$ & $3,30 \pm 0,10 \mathrm{~b}$ & $2,95 \pm 0,04 \mathrm{~b}$ & $78,97 \pm 3,56 \mathrm{a}$ & $14,35 \pm 3,17 \mathrm{~d}$ \\
\hline & & & & & & \\
\hline
\end{tabular}

* Médias seguidas pela mesma letra não diferem entre si pelo teste de Scott-Knott $(p<0,05)$.

Houve diferenças na acidez titulável do iogurte conforme as formulações (Tabela 1). A acidez titulável dos tratamentos com (0:0), (0:2,5) e (0:5), \% de noni e acerola, respectivamente, foi semelhante entre si e superior aos demais tratamentos. E, por sua vez, a acidez dos tratamentos com $(1,25: 1,25)$ e $(2,5: 2,5) \%$ de noni e acerola foi semelhante entre si e significativamente superior as formulações com (2,5:0) e (5:0) \% de noni e acerola que não apresentaram diferenças entre si (Tabela 1). Esse resultado pode ser compreensivo tendo em vista que a polpa de acerola possui acidez titulável elevada $(1,65 \%)$ (MATSUURA et al., 2001) quando comparado a polpa de noni $(0,63 \%)$ (CORREIA et al., 2012).

Valores inferiores aos detectados no presente estudo foram detectados por Schmidt et al. (2012) em iogurtes de polpa de acerola nas concentrações de 2; 4 e 6\%, esses detectaram que a acidez titulável correspondia respectivamente a 0,72; 0,76 e 0,77g de ácido láctico/100g. Ao contrário, Giese et al. (2010), também evidenciaram acidez elevada $(0,835$ a 1,056g de ácido 
láctico/100g) em iogurtes comerciais. Por outro lado, Medeiros et al. (2011) constataram em iogurtes de jaca, acidez de $0,62 \%$ a $0,75 \%$ de ácido láctico.

Apesar das diferenças verificadas entre os iogurtes conforme as formulações, todos apresentaram acidez titulável dentro do estabelecido pela legislação de 0,6 a 1,5g de ácido láctico/100g (BRASIL, 2007).

Para o pH não se verificaram diferenças significativas dos tratamentos (Tabela 1). Todos os iogurtes apresentaram $\mathrm{pH}$ dentro da faixa $(3,5$ a 4,6$)$ aceitável pela legislação brasileira vigente (BRASIL, 2007). Isso pode ter ocorrido naturalmente, devido à formação de sistemas tamponantes das próprias proteínas presentes nas formulações, que estabilizam o meio e dificultam a alteração na concentração de íons hidrogênio, o que caracteriza a variação de pH (SIVIERI \& OLIVEIRA, 2002).

Schmidt et al. (2012) encontraram pH de 4,02 nos iogurtes com 2 e $4 \%$ de polpa de acerola e de 3,99 no iogurte com $6 \%$ da polpa. Giese et al. (2010), ao analisarem iogurtes comerciais observaram $\mathrm{pH}$ até 4,01 , semelhantes aos obtidos neste estudo.

O teor de gordura láctea dos iogurtes variou conforme os tratamentos (Tabela 1). As formulações com $(1,25: 1,25),(0: 5)$ e $(2,5: 2,5) \%$ de noni e acerola não diferiram entre si quanto ao teor de gordura láctea e apresentaram teores inferiores quando comparado aos demais tratamentos. Não obstante, o iogurte com $(0: 2,5) \%$ de noni e acerola apresentou acréscimo de 2,9\% no teor de matéria gorda quando comparado à formulação com (0:5) \% de noni e acerola.

Segundo Costa et al. (2013), o fruto noni possui traços de lipídeos na polpa e nas sementes $(0,37$ e $0,57 \%$, respectivamente), no entanto, o aumento da gordura láctea verificado nas formulações que continham somente noni, que foi de 3,57 e 3,50\% para as formulações com (2,5:0) e (5:0) \% de noni e acerola, respectivamente, possivelmente não está relacionada a esse fato. Outros estudos, na literatura, com iogurtes formulados com polpa de acerola averiguaram teor de gordura láctea em torno de 2,5\% (SCHMIDT et al., 2012), valor inferior quando comparado aos que foram analisados para os iogurtes com $(0: 2,5)$ e $(0: 5) \%$ de noni e acerola $(3,40$ e $3,30 \%$, respectivamente) neste trabalho.

Medeiros et al. (2011) avaliando iogurtes de jaca constataram que estes tinham 2,05\% de lipídeos em sua composição, já no estudo de iogurtes com polpa de tamarindo doce (Tamarindus indica) nas proporções de $4 \%, 6 \%$ e $8 \%$, os valores encontrados para as gorduras totais foram de 2,5\% (MESQUITA et al., 2012), sendo valores inferiores ao mínimo permitido para iogurtes integrais, segundo o Padrão de Identidade e Qualidade para Leites Fermentados (BRASIL, 2007), ficando os valores de gordura compreendidos de 0,6 a 2,9\% para iogurtes parcialmente desnatados. Neste estudo, os valores se encontraram dentro da faixa $(3,0-5,9 \mathrm{~g} / 100 \mathrm{~g})$ estabelecida pela legislação (BRASIL, 2007) para iogurte integral.

O teor de proteínas lácteas dos iogurtes modificou-se conforme as formulações desenvolvidas (Tabela 1), entretanto, os valores se encontraram dentro da faixa (no mínimo 2,9\%) estabelecida pela legislação (BRASIL, 2007). As formulações com $(2,5: 0)$ e (5:0)\% de noni e acerola não diferiram entre si quanto ao teor de proteínas lácteas e apresentaram valores superiores quando comparado aos demais tratamentos.

Segundo Costa et al. (2013), o noni é um fruto que apresenta quantidades significativas de proteínas, variando nas sementes, casca e polpa de 2,64\%; 2,23\%; e 2,24\%, respectivamente. Isso pode explicar a diferença no teor de proteínas dos iogurtes com noni em relação aos demais tratamentos. Em outro estudo, realizado por Medeiros et al. (2011), em iogurte de jaca, esses averiguaram valores de proteínas superiores a $4,8 \%$, atendendo aos critérios estabelecidos pela legislação brasileira, porém superior, quando comparado, aos que foram detectados neste trabalho $(2,94 \%$ a $3,06 \%)$.

Houve diferenças significativas das substâncias fenólicas conforme os tratamentos (Tabela 1). $O$ iogurte com (0:5) \% de noni e acerola apresentou teor de substâncias fenólicas superior $(78,97$ $\mathrm{mg} / 100 \mathrm{~g})$ aos demais tratamentos. Já os tratamentos $(0: 2,5)$ e $(2,5: 2,5)$ apresentaram valores 
significativamente iguais $(69,47$ e $69,76 \mathrm{mg} / 100 \mathrm{~g}$, respectivamente) que, por sua vez, diferiram dos demais tratamentos (0:0), (2,5:0), $(1,25: 1,25)$ e $(5: 0)$, com valores de 56,42; 59,13; 64,69; e 61,04 $\mathrm{mg} / 100 \mathrm{~g}$, respectivamente.

Esta pesquisa corrobora com os dados evidenciados por Canuto et al. (2010) sobre a polpa de noni, cujo teor de fenólicos foi inferior a polpa de acerola, isto possivelmente explica, no presente estudo, as diferenças relevantes de fenólicos nos iogurtes. Haja vista, que o tratamento com $(0: 2,5) \%$ de noni e acerola apresentou teor de fenólicos semelhante ao tratamento $(2,5: 2,5)$ e superiores ao tratamento (2,5:0). Dessa forma, percebeu-se que a concentração de noni não interferiu expressivamente no teor de fenólicos do iogurte que continha as duas polpas de frutas.

De mesma maneira, Vieira et al. (2011) averiguaram que a polpa de acerola, dentre alguns frutos tropicais, destacou-se com $835,25 \pm 32,44 \mathrm{mg} / 100 \mathrm{~g}$ de fenólicos totais, sendo, portanto, a de maior quantidade, seguido pela polpa de caju e de goiaba. Isso explica a alta concentração de compostos fenólicos detectados nos iogurtes formulados com acerola.

Houve efeito de tratamento também para a atividade antioxidante (Tabela 1). O iogurte com $(0: 5) \%$ de noni e acerola obteve o menor valor de $\mathrm{IC}_{50}(14,35 \mathrm{mg} / \mathrm{mL})$, portanto, a maior atividade antioxidante, já que são inversamente proporcionais, porém, não diferiu significativamente do iogurte $(0: 2,5) \%$ de noni e acerola. Esta atividade, expressa em IC ${ }_{50}$, é a concentração do extrato necessária para reduzir $50 \%$ do radical DPPH, em diferentes concentrações. Esse resultado está relacionado, possivelmente, às substâncias presentes na polpa de acerola, como vitamina C (ASSIS et al., 2001) e polifenóis (VIEIRA et al., 2011) que são antioxidantes naturais (KUSKOSKY et al., 2006),

No presente estudo, o iogurte natural (0:0) apresentou $\mathrm{IC}_{50}$ de $139,40 \mathrm{mg} / \mathrm{mL}$, portanto menor atividade antioxidante, seguida pelo iogurte com (2,5:0) \% de noni e acerola (106,33 mg/ $\mathrm{mL})$ e, então, pelos tratamentos $(1,25: 1,25),(5: 0)$ e $(2,5: 2,5)$ sendo 62,$86 ; 56,34 ; 47,61 \mathrm{mg} / \mathrm{mL}$ os respectivos valores de $\mathrm{IC}_{50}$. Apesar disso, os valores de compostos fenólicos do iogurte natural (0:0) e dos tratamentos $(1,25: 1,25)$ e $(5: 0)$ foram estatisticamente iguais.

De maneira geral, percebeu-se que a atividade antioxidante dos iogurtes está diretamente relacionada à concentração de substâncias fenólicas, isto é, quanto maior a quantidade de fenólicos totais, maior a ação antioxidante. Barreto et al. (2009) estudaram 18 polpas de frutos, dentre eles buriti, banana, jaca, carambola, ameixa e nectarina, e concluíram que a atividade anti-radical livre encontrada obteve alta correlação com o teor de compostos fenólicos totais, o que se confirmou no presente estudo.

\subsection{ANÁLISE SENSORIAL}

Houve efeito significativo de tratamento para todos os atributos sensoriais avaliados (consistência, aceitação e intenção de compra), (Tabela 2).

Para a análise sensorial, verificou-se diferenças na consistência dos iogurtes conforme o tratamento (Tabela 2). O iogurte natural (0:0) obteve maior consistência, evidenciada pela maior nota $(6,40)$, sendo semelhante estatisticamente aos iogurtes com $(0: 2,5) \%$ de noni e acerola $(6,13)$ e com $(1,25: 1,25) \%$ de noni e acerola $(6,37)$ e significativamente diferente dos demais tratamentos (Tabela 2). Não obstante, o iogurte (5:0) obteve a menor nota para a consistência $(5,57)$, porém não diferiu estatisticamente das formulações $(2,5: 0),(0: 5)$ e $(2,5: 2,5)$, sendo as notas 5,$79 ; 6,00$ e 5,79, respectivamente. Tal diferença na consistência dos iogurtes pode ser explicada pela adição das polpas, pois em iogurte natural, verificouse a maior consistência, que foi diminuindo com os acréscimos proporcionais de polpa nos mesmos.

Schmidt et al. (2012) em seus estudos sobre a viscosidade de iogurtes com polpa de acerola a 2, 4 e $6 \%$, constataram que a maior viscosidade dos iogurtes ocorreu no tratamento com $2 \%$ de polpa. 


\section{TABELA 2. ANÁLISE SENSORIAL DE SETE FORMULAÇÕES DE IOGURTES EM FUNÇÃO DE DIFERENTES CONCENTRAÇÕES DE NONI (MORINDA CITRIFOLIA L.) E ACEROLA (MALPIGHIA EMARGINATA DC.), MOSSORÓ-RN, 2014.}

\begin{tabular}{cccc}
\hline $\begin{array}{c}\text { Formulação com noni:acerola } \\
(\%)\end{array}$ & Consistência & Aceitação & Intenção de compra \\
\hline$(0: 0)$ & $6,40 \mathrm{a}$ & $7,19 \mathrm{a}$ & $3,95 \mathrm{a}$ \\
\hline$(0: 2,5)$ & $6,13 \mathrm{abc}$ & $7,13 \mathrm{a}$ & $3,97 \mathrm{a}$ \\
\hline$(2,5: 0)$ & $5,79 \mathrm{~cd}$ & $3,95 \mathrm{a}$ \\
\hline$(1,25: 1,25)$ & $6,37 \mathrm{ab}$ & $7,23 \mathrm{a}$ & \\
\hline$(0: 5)$ & & & $4,00 \mathrm{a}$ \\
\hline$(5: 0)$ & $6,00 \mathrm{bcd}$ & $7,08 \mathrm{a}$ & $3,25 \mathrm{~b}$ \\
\hline$(2,5: 2,5)$ & $5,57 \mathrm{~d}$ & $6,22 \mathrm{~b}$ & $3,65 \mathrm{a}$
\end{tabular}

\footnotetext{
* Médias seguidas pela mesma letra não diferem entre si pelo teste de Friedman. Notas e descrições para aceitação: $1=$ desgostei muitíssimo, 2 = desgostei muito, 3 = desgostei moderadamente, 4 = desgostei ligeiramente, 5 = não gostei nem desgostei, 6 = gostei ligeiramente, 7 = gostei moderadamente, $8=$ gostei muito e $9=$ gostei muitíssimo. Notas e descrições para intenção de compra: 1 = certamente não compraria, 2 = provavelmente não compraria, $3=$ tenho dúvida se compraria, 4 = provavelmente compraria e $5=$ certamente compraria.
}

Houve diferenças significativas nas aceitações dos iogurtes conforme o tratamento (Tabela 2). O tratamento (5:0) apresentou a menor nota $(6,22)$, na escala hedônica variando de "gostei ligeiramente" a "gostei moderadamente", diferindo-se significativamente dos demais tratamentos (Tabela 2), que receberam notas próximas a 7,0, correspondendo à "gostei moderadamente" a "gostei muito". Não obstante, as formulações utilizadas apresentaram notas acima de 5,0 (limite inferior de aceitação), o que permite afirmar que os produtos apresentaram características sensorialmente adequadas para a comercialização.

Schmidt et al. (2012), ao desenvolverem formulações de iogurtes com polpa de acerola a $2 \%, 4 \%$ e $6 \%$, averiguaram que a formulação com menor concentração de polpa (2\%) obteve maior aceitabilidade (entre 80 e $96 \%$ ). No atual estudo, verificou-se tal comportamento nas formulações com noni, já as formulações com acerola, independente da concentração, obtiveram, entre si, aceitação semelhante.

Da mesma maneira, houve diferenças significativas na intenção de compra dos iogurtes conforme o tratamento (Tabela 2). O iogurte com (5:0) \% de noni e acerola diferenciou-se significativamente dos demais, e desta maneira, correspondeu na escala hedônica a "tenho dúvida se compraria", enquanto as demais formulações apresentaram-se em "provavelmente compraria" (nota 4,0).

Pelos resultados, evidenciou-se que todos os iogurtes formulados apresentaram-se aptos para comercialização, com nota igual ou acima de 3,0 (Tabela 2). No entanto, as formulações com $(5: 0) \%$ de noni e acerola, seguida de $(2,5: 2,5)$, apresentaram maiores rejeições por parte dos consumidores (Figura 2) e seriam as menos compradas. Em contra partida, as formulações $(0: 2,5)$ e $(2,5: 0)$ apresentaram as menores rejeições e, portanto seriam as mais compradas (Figura 2), embora não tenham diferido estatisticamente $(p<0,05)$ das demais quanto à aceitação (Tabela 2$)$. 
Apesar de não convencionais, a exemplo da acerola e do noni, outros frutos já foram utilizados na elaboração de novos iogurtes, como tamarindo doce (Tamarindus indica) (MESQUITA et al., 2012) e apresentaram boa aceitação quando avaliados sensorialmente, demonstrando potencial para o desenvolvimento dos mesmos, semelhantes aos produtos aqui estudados.

\title{
4 CONCLUSÃO
}

As formulações dos iogurtes com noni e acerola apresentaram diferenças significativas na acidez titulável, gordura láctea e proteínas lácteas, exceto para o $\mathrm{pH}$. Porém todos permaneceram dentro dos padrões de qualidade físico-química estabelecidos pela legislação brasileira.

O iogurte com (0:5) \% de noni e acerola apresentou maior teor de fenólicos totais e junto com o iogurte $(0: 2,5) \%$ de noni e acerola apresentaram maior atividade antioxidante.

A consistência, aceitação e intenção de compra foram influenciadas pelos tratamentos. Com exceção do iogurte(5:0) \% de noni e acerola, todos os demais iogurtes formulados apresentaram aceitação e intenção de compra semelhante ao iogurte natural (0:0).

\section{YOGURTS WITH NONI AND ACEROLA PULP: PHYSICAL AND CHEMICAL PROPRIETES, ANTIOXIDANT ACTIVITY AND SENSORY PROFILE EVALUATION}

\begin{abstract}
The innovation in the development of products by the food industry aims to add differential quality attributes, such as antioxidant properties. Thus, during the yogurt manufacture with new flavors is necessary to do sensory evaluation to ensure a good marketing. Thus, the study aimed to manufacture yogurt from noni and acerola pulp and evaluating the physical and chemical properties, antioxidant activity and sensory profile. The natural yogurt was used as control (0:0) and the other treatments were obtained from noni and acerola pulp proportions: (0:2.5), (2.5:0), (1.25:1.25), (0:5), $(5: 0)$ and $(2.5: 2.5)$. The titratable acidity, milk fat and protein differed with treatment, but remained at according to the quality standards established by the Brazilian legislation. The (0:5) treatment obtained higher phenolic content and together with (0:2.5) treatment showed higher antioxidant activity. Except to (5:0) treatment, other yogurts showed acceptance and purchase intent similar to natural yogurt. The (2.5:0) treatment presented lower rejection by purchase intent test. Thus, all manufactured yogurts show great commercial potential.
\end{abstract}

KEYWORDS: MILK, NONI, PHENOLICS, ACEROLA.

\section{REFERÊNCIAS}

1 ASSIS, S.A.; LIMA, D.C.; OLIVEIRA, O.M.M.F. Activity of pectin methylesterase, pectin content and vitamin C in acerola fruit at various stages of fruit development. Food Chemistry, v. 74, p. 133-137, 2001.

2 BARRETO, G.P.M.; BENASSI, M.T.; MERCADANTE, A.Z. Bioactive compounds from several tropical fruits and correlation by multivariate analysis to free radical scavenger activity. Journal of the Brazilian Chemical Society, v. 20, n. 10, p. 1856-1861, 2009.

3 BRASIL. Ministério da Agricultura, Pecuária e Abastecimento. Secretaria de defesa agropecuária. Departamento de inspeção de produtos de origem animal. Instrução Normativa n. 46, de 23 de outubro de 2007. Regulamento Técnico de Identidade e Qualidade (RTIQ) de Leites Fermentados. Diário Oficial da União, p. 5, 24/10/2007. Seção 1. 
4 CANUTO, G.A.B.; XAVIER, A.A.O.; NEVES, L.C.; BENASSI, M.T. Caracterização físico-química de polpas de frutos da Amazônia e sua correlação com a atividade anti-radical livre. Revista Brasileira de Fruticultura, Jaboticabal, v. 32, n. 4 , p. $1198-1205,2010$.

5 CORREIA, A.A.S; GONZAGA, M.; AQUINO, A.; SOUZA, P.; FIGUEIREDO, R.; MAIA, G. Caracterização química e físico-química da polpa do noni (Morinda citrifolia L.) cultivado no estado do Ceará. Alimentos e Nutrição, Araraquara, v.22, n.4, p. 609-615, 2012.

6 COSTA, A.B.; OLIVEIRA, A.M.C.; SILVA, A.M.O.; MANCINI FILHO, J.M.; LIMA, A. Atividade antioxidante da polpa, casca e sementes do noni (Morinda citrifolia Linn). Revista Brasileira de Fruticultura, v. 35, n. 2, p. 345-354, 2013.

7 ESCARPA, A.; GONZÁLEZ, M.C. An overview of analytical chemistry of phenolic compounds in foods. Critical Reviews in Analytical Chemistry, v. 31, n. 2, p. 57-139, 2001.

8 FENNEMA, O.R. Química de los alimentos, 2a . Edição, Zaragoza: Acríbia, Espanha, 2000. 1258p.

9 FERREIRA, D.F. Programa de análises estatísticas (statistical Analysis sotware) e planejamento de experimentos SISVAR 5.0 (Build 67). Lavras: DEX/UFLA, 2003.

10 GIESE, S; COELHO, S.R.M; TÉO, C.R.P.A; NÓBREGA, L.H.P; CHRIST, D. Caracterização físico-química e sensorial de iogurtes comercializados na região oeste do Paraná. Revista Varia Scientia Agrárias, vol. 01, n. 01, p. 121-129, 2010.

11 INSTITUTO ADOLFO LUTZ. Normas Técnicas do Instituto Adolfo Lutz: métodos químicos e físicos para análise de alimentos. 4 ed. São Paulo, 2008, v.1, 1020p.

12 ISHIKAWA, T.; SHIGEOKA, S. Recent advances in ascorbate biosynthesis and the physiological significance of ascorbate peroxidase in photosynthesizing organisms. Bioscience, biotechnology and biochemistry, v. 72 , n. 5 , p. 1143-1154, 2008.

13 KUSKOSKI, E.; ASUERO, A.; MORALES, M.; FETT, R. Frutos tropicais silvestres e polpas de frutas congeladas: atividade antioxidante, polifenóis e antocianinas. Ciência Rural, v. 36, n. 4, p. 1283-1287, 2006.

14 LIMA, G.A.L.V.; MELO, A.E.; MACEL, S.I.M.; LIMA, S.E.D. Avaliação do teor de antocianinas em polpa de acerola congelada proveniente de fruto de 12 diferentes aceroleiras. Ciência e Tecnologia de Alimentos. Campinas, v. 23, n. 1, p. 101-103, 2003.

15 LOURES, M.M.R.; MINIM, V.P.R.; CERESINO, E.B.; CARNEIRO, R.C.; MINIM, L.A. Análise descritiva por ordenação na caracterização sensorial de iogurte diet sabor morango enriquecido com concentrado protéico de soro. Ciências Agrárias, Londrina, v.31, n.3, p.661-668, 2010.

16 MATSUURA, F.C.A.U.; CARDOSO, R.L.; FOLEGATTI, M.D.S.; OLIVEIRA, J.R.P.; OLIVEIRA, J.D.; SANTOS, D.D. Avaliações físico-químicas em frutos de diferentes genótipos de acerola (Malpighia punicifolia L.). Revista Brasileira de Fruticultura, Jaboticabal, v.23, n.3, p. 602-606, 2001.

17 MEDA, A.; LAMIEN, C.E.; ROMITO, M.; MILLOGO, J.; NACOULMA, O.G. Determination of the total phenolic, flavonoid and proline contents in Burkin Fasan honey, as well as their radical scavenging activity. Food Chemistry 2005, 91(3), 571-577.

18 MEDEIROS, T.C.; MOURA, A.S.; ARAÚJO, K.B.; AQUINO, L.C.L. Elaboração de iogurte de jaca: Avaliação físicoquímica, microbiológica e sensorial. Scientia Plena, v. 7, n. 9, p.1-4, 2011.

19 MESQUITA, R.V.S.C.; FIGUEIREDO NETO, A.; TEIXEIRA, F.; SILVA, V.O. Elaboração, análise físico-química e aceitação do iogurte com adição do tamarindo "doce" (Tamarindus indica L.). Revista Brasileira de Produtos Agroindustriais, v. 14 , p. $381-387,2012$.

20 NIELSEN. The Nielsen Company. Resultados do consumo no Brasil em 2007. Disponível em: <http://br.nielsen.com/ news/fechamento2007.shtml. Acessado em 15/02/2013.

21 NIELSEN. The Nielsen Company. Resultados do consumo no Brasil em 2011. Disponível em: <http://br.nielsen.com/ news/fechamento2011.shtml. Acessado em 27/02/2013. ORDÓÑEZ, J.A. Tecnologia de alimentos - Alimentos de origem animal, v. 2. Porto Alegre, RS: Artmed, 2005, 279p.

23 SCHMIDT, C.A.P.; PEREIRA, C.; ANJOS, G.; LUCAS, S.D.M. Formulação e avaliação sensorial hedônica de iogurte com polpa de acerola. Revista Eletrônica Científica Inovação e Tecnologia, v.1, n.5, p.10-14, 2012.

24 SINGLETON, V.L.; ROSSI, J.A. Colorimetry of total phenolics with phosphomolybdic-phosphotungsticacid reagentes. American Journal of Enology and Viticulture. v.16, p. 144-158, 1965. 
25 SIVIERI, K.; OLIVEIRA, M.N. Avaliação da vida de prateleira de bebidas lácteas preparadas com "fatreplacers" (Litesse e Dairy-Lo). Ciência e Tecnologia de Alimentos, v. 22, n. 1, p. 24-31, 2002.

26 THAMER, K.G.; PENNA, A.L.B. Caracterização de bebidas lácteas funcionais fermentadas por probióticos e acrescidas de prebióticos. Ciência e Tecnologia de Alimentos, v. 26, n. 3, p. 589-595, 2006.

27 VIEIRA, L.M.; SOUSA, M.S.B.; MANCINI-FILHO, J.; LIMA, A. Fenólicos totais e capacidade antioxidante in vitro de polpas de frutos tropicais. Revista Brasileira de Fruticultura. Jaboticabal - SP, v. 33, n. 3, p. 888-897, 2011. 